\title{
舴- \\ Dilemas en la investigación de migraciones clandestinas
}

\author{
Chabier Gimeno-Monterde \\ Departamento de Psicología y Sociología \\ UNIVERSIDAD DE ZARAGOZA. \\ CHABIERG@UNIZAR.ES
}

Resumen: La investigación de las migraciones clandestinas desde el Trabajo Social presenta dificultades y riesgos, relacionados a menudo con la posición del investigador. Quienes tienen una posición académica o externa a quienes protagonizar estos itinerarios migratorios apenas vislumbran qué hay en ellos, pudiendo caer en la victimización o la criminalización.

Mientras que los profesionales del Trabajo Social que intervienen a diario con los migrantes pueden encontrarse con un dilema. Saber más sobre quienes entran irregularmente en un Estado. O evitar saber, administrativizando su relación técnica y personal con el migrante.

La experiencia presentada aquí opta metodológicamente por permanecer al lado de los migrantes y no en frente; por saber y difundir lo sabido sobre las migraciones clandestinas de menores no acompañados. Reflexionando también sobre los riesgos éticos de estas 
investigaciones, así como sobre los procedimientos a los que recurrimos para saber.

Palabras clave: menores no acompañados, protección de menores, migración irregular, ética, metodología.

\section{Dilemmas in research on illegal migration}

Abstract: Research on clandestine or illegal migration with respect to social work is fraught with difficulties and risks that are often associated with the position of the researcher. Those who hold an academic post or one that is external to the key players involved in these migrant routes rarely see what they entail, and may well settle for victimisation or criminalisation.

Whereas professional social workers who are involved on a daily basis with migrants may be confronted with a dilemma: either to find out more about those that enter a country illegally. or else to avoid finding out, relegating their technical and personal relations with the migrant to a purely administrative capacity.

The experience presented here adopts a methodological decision to remain with the migrants rather than cross over to the opposite side; to find out and disseminate the knowledge gathered on the illegal migration of unaccompanied minors. Also reflecting on the ethical risks of such research, as well as the procedures we resorted to in order to find out such information.

Keywords: unaccompanied minors, protection of minors, illegal migration, ethics, methodology. 


\section{Dilemas en la investigación de migraciones clandestinas}

Chabier

Gimeno-Monterde

\section{MENORES QUE MIGRAN SOLOS}

Los denominados "menores extranjeros no acompañados" son aquellos jóvenes menores de edad que migran solos e irregularmente de las áreas pobres del Planeta a las más ricas. En el caso europeo, la legislación de los diversos Estados de la Unión ampara la acogida de estos adolescentes en los sistemas de protección de menores, hasta que alcanzan la edad adulta (Quiroga, 2005).

Tras la migración de miles de menores africanos a través de esta grieta legislativa en la frontera (Jiménez, 2011: 104), su acogida institucionalizada es objeto de debate en toda Europa. En España hasta el punto de cuestionar si las Comunidades Autónomas no habrán recibido competencias explícitas e implícitas en la política migratoria estatal. Explícitamente se les reclamaría que protegieran a estos jóvenes, mientras implícitamente se les responsabilizaría de evitar el efecto llamada que dicha acogida supone (Gimeno, 2013c: 110).

En esa misma línea, diversos informes emitidos por instituciones públicas, que tutelan a estos menores o que velan por sus derechos, se refieren a menudo a un grupo homogéneo de jóvenes, que son "conscientes" de su estatus legal de desamparo, y que han emigrado a Europa "para ser atendidos" por las administraciones locales (Justicia de Aragón, 2007: 122). Sin embargo, somos cada vez más los investigadores que hablamos de 
un colectivo de menores mucho más heterogéneo. Pues entendemos que, más allá de los adolescentes acogidos por las instituciones, hay otras vías de migración que permanecen ocultas, por voluntad de sus actores o, en ocasiones, por negligencia del Estado. De manera que el itinerario estandarizado por los Servicios de Protección autonómicos para atender a los menores no acompañados es sólo uno de los recorridos por éstos (Gimeno, 2013e).

Esa disensión, esas investigaciones que han dado lugar al cuestionamiento de los discursos institucionales, parten de antropólogas, educadoras, sociólogas y trabajadoras sociales que intervienen profesionalmente con los jóvenes migrantes. Ahí están las aportaciones de Núria Empez, Elísabet Marco, Ainhoa Rodríguez y Mercedes Jiménez entre otras. Compartiendo con ellas este proceso, y a la vista de este contexto migratorio, en la reflexión que aquí presentamos tenemos como objetivos evidenciar, por un lado, las dificultades para investigar las migraciones juveniles clandestinas. Y por otro, los conflictos técnicos y éticos que nuestra doble condición de profesionales e investigadores pueden provocar en el Trabajo Social.

\section{SÍNTESIS DE UNA INVESTIGACIÓN}

La investigación doctoral de la que nace esta reflexión se ha presentado bajo el título "Como una bolisma. Menores que migran solos a Aragón"1, y ha sido desarrollada en las tres capitales provinciales aragonesas, donde estos menores han sido acogidos por el gobierno autónomo.

Entre 2007 y 2012 hemos realizado un trabajo de campo que ha incluido dieciséis entrevistas semiestructuradas a jóvenes migrantes y treinta y nueve a técnicos de administraciones públicas o entidades privadas. En este periodo el autor, trabajador social y sociólogo, ha realizado también observación participante en los proyectos educativos en los que intervenía con estos menores, además de en otros espacios urbanos donde concurren jóvenes migrantes.

${ }^{1}$ Dirigida por los profesores Carlos Gómez Bahillo y Aurora López Azcona, de la Universidad de Zaragoza, a quienes muestro desde aquí mi agradecimiento. 
En cuanto al análisis documental sobre esta migración, éste ha incluido los informes anuales sobre menores realizados por el defensor del pueblo autonómico (Justicia de Aragón), así como otros emitidos por o para el Instituto Aragonés de Servicios Sociales (responsable del Servicio de Protección de Menores) y por entidades sociales que trabajan con jóvenes migrantes.

Y, como complemento cuantitativo, se analizó la información anonimizada sobre los 298 expedientes de tutela administrativa de los menores no acompañados acogidos entre enero de 2000 y diciembre de 2010 por el Instituto Aragonés de Servicios Sociales (IASS). Estos datos se trataron estadísticamente y los resultados nos muestran un colectivo de adolescentes, entre 15 y 17 años de edad, procedentes del Magreb en un 78'5 \% y del África Occidental en un $17^{\prime} 4 \%$. De entre ellos apenas un $4^{\prime} 4 \%$ son niñas (Gimeno, 2013d).

\section{INTERVENCIÓN, SABERES Y ÉTICAS}

"Bueno cuidate mutxo xavi ,y sobre las entrevistas y eso, tu has loke ves $k$ es algo bueno,y que dios estara contigo, insha'alah" (joven árabe magrebi).

Como primer paso en nuestra reflexión sobre la posición del Trabajo Social ante esta migración irregular hemos concluido que las políticas migratorias dirigidas a los menores solos son percibidas por éstos como un ejercicio de Poder u Orden, entendido éste como la búsqueda de uniformidad, de estabilidad (Gimeno, 2013c: 111). Y que, al mismo tiempo, los menores que migran solos suponen para el Estado un Desorden, al haber accedido a su territorio por una 'grieta' en la legislación, que obliga a su protección. Como consecuencia, los dispositivos de control migratorio han reaccionado intentando disciplinar jurídicamente este flujo mediante las leyes y uniformar a los menores mediante el Trabajo Social, anulando progresivamente su capacidad de subvertir el Orden al burlar las fronteras. La resistencia anudada de los menores ha sido buscar nuevas maneras de eludir ese control, bien migratorio, bien del Trabajo Social. Configurándose así como 'Gente': algo impredecible y que se aleja constantemente del equilibrio (Bergua, 2007: 40). 
En una primera etapa de los estudios sobre las migraciones de jóvenes solos, el intento de crear perfiles, de uniformar y de estabilizar ha respondido a ese Orden (Unicef, 2009; Quiroga, 2010; Cabrera, 2005). Con todo, estas aproximaciones desde lo institucional dejan fuera todo lo que algunos investigadores percibíamos como inestable desde la práctica profesional, pues generaba conflictividad y tensiones entre Administración, trabajadores sociales y menores. Ese Desorden ha sido determinante también en el diseño metodológico de nuestra investigación.

Eludir una investigación que orbitase en torno al Orden ha implicado también, desde el inicio de la investigación, algunos riesgos éticos. Estos riesgos, como en el caso de las otras investigadoras que nos sirven de referencia, señalan hacia lo que no se puede o no se quiere saber: todo lo referente a esta movilidad que los "especialistas de la acción" (técnicos y políticos) puedan utilizar para predecir y estabilizar este flujo tan complejo.

De tal manera que, la información que nuestra praxis profesional nos aportaría como plus de conocimiento del campo, puede devenir en un plus de descubrimiento de lo oculto para quienes pretenden el control migratorio desde las instituciones estatales ${ }^{2}$. Esta posibilidad nos sitúa a menudo como investigadores en una condición de agentes dobles, y como profesionales en una posición de ilegitimidad moral para compartir lo sabido.

"Discutimos durante todo el trayecto en taxi por la periferia de Tánger [Marruecos] sobre las estrategias de ocultación y burla que utilizan los chavales. [Educadora] cree que no puedo describirlas, pues pongo en peligro esas vías para eludir el control fronterizo y los límites de la protección de menores" (cuaderno de campo, marzo 2012).

No hemos encontrado testimonios, ni personales ni académicos, sobre la ausencia de este riesgo. Es decir, sobre la voluntad explícita de usar lo sabido para facilitar el control al Poder. Lo cual ya dice mucho sobre la implicación de las Ciencias Sociales en cuanto a los grandes relatos de lo migratorio. Pero sí es cierto que para una parte de los que investigan esta migración, sólo los

2 "I must be very meticulous with the kind of information I might give in articles or reports, making sure that I will not harm them, or put them in danger" (Empez, 2009: 165). 
próximos a la intervención están conociéndola con más matices y riqueza. Mientras que los que lo hacen como externos, o bien se ven condicionados por su posición y redundan en las obviedades y las ideologías (humanitarias, demócratas, garantistas, etc), o bien reproducen ciertas lógicas entristas o paracaidistas propias de algunas antropologías postcoloniales y otros movimientos recuperadores -en el sentido del término que le otorga el situacionismo contemporáneo.

Otro de los mayores riesgos éticos de estas "etnografías" sería el inevitable etnocentrismo. Para Bergua (2011: 169) el mecanismo básico de autoinstitución de las sociedades es la expulsión simbólica de los otros fuera de la cultura, considerando como tal la propia. Ahora bien, la cultura occidental o judeocristiana se autoinstituye frente a las otras, pero al mismo tiempo "dice" proclamar la unión de toda la especie humana. Este "humanismo", creemos, subyace en el fondo de muchas metodologías con las que algunas investigaciones pretenden salvar la posición que como externos tenemos en la migración de los jóvenes solos. Este pretendido universalismo no sería entonces sino otra versión etnocentrista, que parte del afán occidental de "comprender a los otros". Cuando para los menores solos, y para otros tantos Otros, el reconocimiento de la diferencia entre investigadores y jóvenes es lo único concebible, amén de la curiosidad natural. Asumir esta limitación ha sido otra premisa metodológica de nuestra investigación.

Con todo, esto no ha evitado que viviéramos las contradicciones propias de unos roles cambiantes entre la investigación y el Trabajo Social. En algunas ocasiones los menores obviaban la posibilidad de establecer una relación horizontal, pero intentaban introducir una hiperreflexividad ${ }^{3}$ perversa (pidiendo dinero para gastos extraordinarios, etc), que los acercaba a una victimización muy útil con otros técnicos. En otros casos, los menores que conocíamos en el ámbito profesional nos advertían de que, en cuanto a nuestro rol de investigadores, preferían ocultar algunas informaciones. De hecho, el ocultamiento de lo vergonzoso y el progresivo debilitamiento de las barreras con el paso del tiempo siempre han estado ahí (Jiménez, 2011: 37). Es más, en muchas

3 "Cuando un sujeto sabe lo que el otro cree que espera de él" (Bergua, 2011: 195). 
ocasiones, nuestras actuaciones y discursos adecuados al rol del profesional que pretendía eludir el encuadramiento homogéneo en el Trabajo Social, también nos han obligado a no olvidar que el objeto y el sujeto se están construyendo mutuamente en esa relación (Monteros, 2007: 60). En estas y en otras ocasiones, el investigador-profesional ha tenido que asumir una reflexividad vigilante (Jiménez, 2011: 274).

\section{SABERES Y MOTIVACIONES}

Ahora bien, la consideración de estos riesgos éticos y metodológicos no ha derivado en una parálisis. Al contrario, han motivado una búsqueda del sentido primigenio del impulso que inició nuestra investigación: la curiosidad innata del investigador, cuando además es un trabajador en lo social. El goce de conocer mejor lo que se intuye en la práctica cotidiana. El placer que transmiten las confidencias y complicidades que los menores comparten cuando se traspasan los códigos profesionales de un Trabajo Social instituido como control migratorio (Gimeno, 2013b).

Un placer que, para todo curioso, conlleva una necesidad de alparciar ${ }^{4}$, de continuar con el chatting. Esta conversación ha constituido una de las maneras de vivir con los menores su proceso migratorio. Siguiendo a Virno (2003a: 36), la "productividad de la charla" consiste en la autonomía de esta respecto a los fines predefinidos y a la necesidad de reproducir fielmente la realidad: "la charla no representa nada, pero precisamente por ello puede producir de todo". Esta ha sido una de las puertas al imaginario migratorio de los menores que hemos explorado durante mucho tiempo.

También, cuando el investigador se sitúa al margen de los objetivos explícitos de control, existe la necesidad de mostrar, o en este caso de "demostrar" que las fronteras administrativas, como todo lo que se pretende estable, son débiles y penetrables. Y, por lo tanto, sus diseñadores técnicos son burlables. Por extensión,

${ }^{4}$ En aragonés "cotillear, chismorrear". Pero también, más allá de las pobres traducciones entre lenguas, alparciar como forma tradicional de transmitir la información fuera de los canales públicos u oficiales. Como vehiculización del saber de la Gente. 
la falibilidad del Trabajo Social en las sociedades de control (Deleuze, 1999) alienta a quienes vivimos esta disciplina como una vocación maleada por el capitalismo. Así, nuestra investigación no oculta que al acercarnos así a la Gente pretendemos que el pensamiento único entre los profesionales de lo social se vea cuestionado. Pues aunque los que mandan creen que saben sobre los mandados y que pueden predecirlos, en realidad, aun siendo útil ese saber, el Orden nunca sabe mucho sobre las alteridades (Bergua, 2011: 198).

¿Esto nos convierte a quienes analizamos así al Trabajo Social en críticos? Aquí concluimos que no cabe esta autodefinición como sujetos ante los que la Administración estaría alerta (Empez, 2009: 165). Más bien, asume que vivimos en ese pluriverso (y no uni-verso) sin centro, en el que no sabemos nada de los Otros, aunque diariamente trabajemos con ellos. Por lo que nuestra información tampoco será determinante. Y los intentos estatales de predecir utilizándola serán permanentemente fallidos.

Hay otros saberes, hay otras praxis y, como todo lo instituyente, están también en el corazón de nuestras rutinas institucionalizadas. Por ello pretendemos ir, cuando sea posible, más allá de la actual heteronomía de las disciplinas de lo social. Mientras asumamos que las leyes que nos acotan y "gobiernan a distancia" (Monteros, 2007: 370) tienen un origen extrasocial, como si no fuera obra de humanos, no comprenderemos nuestra capacidad instituyente como colectivo profesional. Nuestra potencial autonomía, que no será una autonomía en la praxis individual si no coincide con la autonomía del conjunto. Siguiendo a Castoriadis (1983), creemos que esa autonomía implica asumir que son los integrantes de una sociedad los que dan a las instituciones el poder que tienen. La consiguiente ruptura haría surgir del "magma simbólico" el cuestionamiento del Orden, de lo uniforme.

En ese marco, los intensos debates sobre qué decir o no decir sobre lo que no se sabe, tienen una posible salida. Reconocer que en la investigación que presentamos, como en otras que hemos conocido desde sus entrañas, los buscavidas y sus prioridades se muestran en aquello que no los implica como sujetos de control futuro, sino como "surferos" de olas ya pasadas. Conscientes de que las falsificaciones documentales, cambios de identidad y 
otras tácticas concretas nacen con caducidad previsible, ante un Estado que obliga a las resistencias a reanudarse continuamente (Bergua, 2007: 35).

Las grietas de la frontera se señalan en nuestras investigaciones, casi siempre, cuando ya han sido reparadas. Pero el deleite al contemplarlas cumple ese objetivo fijado por Bauman de "ayudar a que las personas comunes encuentren su sendero en el laberinto" 5 .

\section{TÉCNICAS DE APROXIMACIÓN}

Partiendo de esa inestabilidad e intratabilidad de los menores que migran irregularmente, las técnicas que hemos utilizado debían abarcar también esa mano abierta a lo no sabido desde lo institucional, ampliando así el acceso a lo sabido a causa de la praxis profesional.

Al saber clásico nos hemos acercado a través de técnicas comunes en otros estudios sobre las migraciones juveniles. En primer lugar, a través de la captación y análisis de datos cuantitativos ${ }^{6}$. Mientras, en paralelo, se realizó una labor de captación y estudio de las fuentes secundarias que han informado teórica y descriptivamente la investigación, atendiendo especialmente a lo generado desde o para las instituciones, muchas de ellas con competencias en protección de la infancia.

El acceso a los discursos que luego se han entrelazado como textos en el informe final de la investigación se llevó a cabo mediante entrevistas a jóvenes y técnicos que intervenían con ellos. Las entrevistas concertadas con los jóvenes migrantes han seguido el principio de intentar representar a los dos orígenes mayoritarios en los expedientes: los magrebís y los subsaharianos del África Occidental. Así como un universo de sentido, por lo que la muestra se ha construido no de forma aleatoria, sino intencionalmente (Bergua, 2011: 123).

A los jóvenes se ha llegado, además de por los contactos establecidos durante la práctica profesional, por la mediación de algunas entidades sociales. A través de éstas se ha podido

\footnotetext{
${ }^{5}$ Entrevista al sociólogo polaco en La Nación (15-2-2006).

${ }^{6}$ Sobre el proceso de análisis cuantitativo, véase Gimeno (2013d).
} 
entrevistar a jóvenes solos con trayectorias migratorias que la Administración evalúa como exitosas, así como a algún afectado por medidas judiciales. Estas entrevistas se han realizado en entornos institucionales, como sedes de proyectos sociales o recursos de acogida. Sin embargo, las otras han tenido escenarios propios de la relación previa con los propios entrevistados o con otros jóvenes que les remitían a nuestra investigación, siguiendo el efecto de "bola de nieve": cafeterías, kebabs, esquinas donde se traficaba, parques y terrazas. En todas las entrevistas hemos obviado el principio de que en una investigación que proviene de la práctica profesional "es importante no dejarse influir por los contactos previos" (Bergua, 2011: 163). Al contrario, la investigación no ha querido eludir a jóvenes con los que el contacto ha sido, en algunos casos, biográficamente muy extenso.

En todos los casos fueron entrevistas semiestructuradas, donde el guion que las orientaba era conocido previamente por los jóvenes, que han introducido en la conversación todo aquello que han querido expresar al margen de los temas ya fijados. Se ha intentado así romper con el rol profesional de "educador", con el fin de enarbolar otro de narrador, casi de cuentista. Teniendo en cuenta que el entrevistado intenta informarse de lo que el entrevistado sabe para valorar lo que le cuenta, y que el relato de una historia supone para el entrevistado una racionalización y puesta en orden de su vida (Bergua, 2011: 135 y 163). Conscientes, en suma, de que los jóvenes querían contar algo y de que esa transmisión de saber les obligaba a rastrear en su memoria y a evaluar su trayectoria migratoria, hemos respetado en todo momento el ritmo narrativo de las entrevistas.

A mayor relación con el joven por nuestra práctica profesional, los campos abarcados y su profundidad han sido mayores también, lo que ha permitido buscar diferencias discursivas asociadas a la posición social de los jóvenes (Bergua, 2011: 132). De ahí que a la información recabada y analizada posteriormente en las entrevistas, hemos unido la proveniente de los cuadernos de campo, donde estos menores eran una referencia común.

Antes de referirnos a estos cuadernos, es preciso reseñar el otro bloque de entrevistas, éstas más estructuradas, el dedicado a los profesionales que han intervenido con los menores solos. Se trata de un nutrido grupo de técnicos que abarcan la 
Administración pública aragonesa y estatal, y las entidades privadas que participan en la acogida o reciben extutelados que salen de la misma.

Pero también ha habido entrevistas, todas ellas no grabadas por voluntad de los profesionales, realizadas de forma anónima a informantes cualificados. En este caso, se trataba de antiguos trabajadores del sector público y del privado; de trabajadores en activo que preferían no ser identificados como informantes en sus puestos de trabajo; así como de activistas sociales relacionados con los derechos de la infancia y la juventud.

"Las oenegés estamos muy limitadas a denunciar ese tipo de cosas, si luego Menores no ha tomado cartas en el asunto" (profesional de entidad privada).

Estas entrevistas se han grabado en los puestos de trabajo, o bien, si eran anónimas, en cafeterías y otros lugares donde era viable eludir el silencio instituido. Esta dinámica de visibilidad e invisibilidad de los técnicos no ha sido una premisa previa de la investigación. Pero se ha impuesto al advertir algunos de los entrevistados de la ausencia de algunos discursos en nuestros primeros textos y del riesgo de institucionalización de nuestra investigación, si no se accedía a condiciones y lugares donde lo sabido podía ser explicitado. Algo que se puede ilustrar con la negativa de algunos técnicos a ser grabados, sufriendo la información que han facilitado el filtro de las anotaciones del investigador.

Por su parte, los cuadernos de campo han recogido en formato escrito notas, así como fotocopias de sentencias $u$ otros documentos, fotografías, enlaces en internet y muchas otras referencias. Siempre sin una planificación previa, a no ser la voluntad de fijar la memoria de la práctica del Trabajo Social en contacto con estos y otros jóvenes. Se trata, por tanto, de una fuente no estructurada, donde la información consignada carecería de valor para agentes ajenos a la doble posición del investigador.

\section{FROTAR Y COMPARTIR: EL MAGNETISMO}

Y es que con las técnicas más clásicas, comunes a otras investigaciones sobre esta migración irregular, apenas nos 
aproximábamos a lo reflejado en los cuadernos de campo, en los que constatábamos desde el principio de la investigación la incapacidad del Trabajo Social para anticiparse al devenir de los jóvenes que migran solos. Ellos mismos lo perciben así.

"Te preguntan. Te quieren sacar algo. Piensan que la gente (es) tonta" (joven árabe magrebí).

"Se pasaron mucho tiempo pidiéndome el número de teléfono de mi padre, pero no sacaron nada de mi" (joven bereber magrebí).

Y si los técnicos y los políticos apenas perciben nada es por su posición. Una posición que condiciona su acceso al imaginario de estos adolescentes, situando la victimización o la criminalización de esta migración como un filtro que ancla a los profesionales en un punto ciego.

Sin embargo, el imaginario de estos jóvenes globales, al que accedíamos diariamente durante la práctica profesional, se nutre, como ocurre con los jóvenes nativos, del consumismo y del hedonismo, extensamente enraizados en su campo social transnacional (Suárez, 2008). Para Maffesoli (2004: 109), el carpe diem y otras expresiones de la actual expansión de lo existencial son contrarias a las ideologías y acciones en las que todavía se mueven los políticos, "trabajadores sociales" y otros poderes públicos. Y muestran una "cierta reticencia antropológica hacia todos los poderes, que no deja de expresarse puntualmente con mayor o menor eficacia según el tiempo y el lugar". De forma que, para alejarnos de las estructuras simbólicas del Orden, habíamos de situarnos fuera del Trabajo Social, allá donde éste no ve: algo que ya hacíamos, conviviendo on line con ellos. Durante la práctica profesional de la que nace la investigación se generaron perfiles virtuales, en las redes sociales Tuenti y Facebook. A través de ellas y de otras herramientas como los portales Youtube o Myspace, hemos interactuado durante varios años con jóvenes tutelados, ex-tutelados y trabajadores de lo social. Por estos canales se ha difundido igualmente la existencia de la investigación entre los menores y sus iguales, posibilitando también la concertación de entrevistas (la ya citada "la bola de nieve").

Las redes sociales virtuales nos han mostrado un magma simbólico que no cabe dentro de los procedimientos homogéneos de 
inserción en la sociedad de acogida, tal y como son aplicados por el Trabajo Social: más bien, nos aclaran que muchos jóvenes eluden el enfrentamiento con los sistemas de protección, optando por el éxodo o la deserción simbólica e intersticial (Virno, 2003b: 72). Y es que, como todos los simbolismos, el universo simbólico que se pretende imponer a los menores solos acogidos para uniformarlos (basado en la formación académica y la inserción laboral) tiene consecuencias no previstas. Los simbolismos no responden a un orden racional, y están llenos de intersticios y de grados de libertad (Castoriadis, 1983), en los que caben la rabia contra el Estado, la ocultación de los deseos migratorios no asumibles por el Trabajo Social victimizador y un largo etcétera (Gimeno, 2013a).

Evidentemente, la navegación on line no siempre ha llegado a buen puerto y está salpicada de negativas a "agregarse", dudas, contrastes de las fuentes y otras acciones de prospección propias de una investigación que ha convivido tanto on como off line; pero también de una migración que se mueve dentro y fuera de "lo visible". El factor "azar" o la "oportunidad" (Empez, 2009: 163) forman parte de esta doble vía entre la cibervida y la vida analógica. De hecho, las observaciones más interesantes surgen casi siempre de lo inesperado, más allá de los guiones formales de la investigación (Mateo, 2010: 20).

De forma similar, la voluntad de llevar más allá del momento vivido una memoria de la praxis diaria, propia de los cuadernos de campo, ha vertebrado también otras dos técnicas que deben considerarse como referencias de la investigación y con las que hemos querido experimentar y, cuando ha sido posible, innovar: la observación participante y la deriva.

La deriva o el arte de deambular, surgida dentro del movimiento situacionista (Debord, $1958: 20$ ), ha sido un experimento más dentro del intento de romper con la visión instituida que, incluso en los discursos de los jóvenes, pugnaba por imponerse en el texto resultante de la investigación. La intuición nacida en ciertos momentos del Trabajo Social que ejercíamos indicaba la necesidad de dejarse llevar por los espacios urbanos de la ciudad de Zaragoza donde los menores han convivido con sus iguales, con los técnicos o con otros actores de su migración. Deambular con el fin de captar las esencias psicogeográficas en 
unos espacios finalmente recurrentes, y quizá sobreescritos por el paso de varias etapas de este flujo migratorio.

En esta dérive hemos podido captar cómo para estos jóvenes la ciudad, como habrá ocurrido en otras donde se hayan asentado los "no acompañados", se concibe también como un paisaje psíquico donde predominan los huecos. Esto es, hay partes, aquellas que son inquietantes para lo institucional o para otros actores de lo instituido, que son "olvidadas"; mientras los jóvenes que migran solos, con sus relaciones endogámicas, unas veces, o sus relaciones de prospección, otras, han construido infinitas ciudades posibles en esos vacíos resultantes ${ }^{7}$.

Las fotografías en los perfiles de las redes sociales virtuales, donde hemos ampliado la deriva a la vida on line, así como las realizadas por el investigador en las derivas urbanas, han acabado descubriendo nuevos ámbitos de presencia de los menores solos, así como la universalidad de su deseo de dejar un rastro. De superar su desechabilidad, ampliando los términos de Bauman (2005: 24).

Estos espacios urbanos deambulados se situaban en el Casco Viejo zaragozano, tanto en su zona degradada como en la de ocio juvenil nocturno, y en las proximidades de algunos centros de acogida en la misma ciudad.

Y, finalmente, llevando más allá la experimentación, hemos querido también moldear nuestra observación participante. Si atendemos a la definición clásica, esta técnica emblemática de la antropología consiste en investigar una colectividad, elegida según los objetivos que dicta un plan de investigación y participando en ella como un miembro más, con el fin de conocerla en su dimensión subjetiva (Bergua, 2011: 165). Sin embargo, y en conexión con el debate sobre la ética de la investigación cuando ésta se comparte con la práctica profesional en lo social, esta técnica no ha sido desarrollada, como ya se advertía, siguiendo un modelo entrista. Al contrario, coincidiendo con otras investigadoras, la

${ }^{7}$ Coincidimos así con Deleuze (2000: 383), cuando dice que "la vida del nómada es intermezzo. (...) Por más que el trayecto nómada siga pistas o caminos habituales, su función no es la del camino sedentario, que consiste en distribuir a los hombres en un espacio cerrado, asignando a cada uno su parte y regulando la comunicación entre las partes. El trayecto nómada hace lo contrario, distribuye los hombres (...) en un espacio abierto, indefinido". 
intervención como actor del Trabajo Social no es una táctica sino una opción ética y moral ${ }^{8}$.

A partir del momento en que la curiosidad primigenia dio paso a un deseo crecientemente estructurado de saber, en un sentido no clásico del verbo, la observación transcendió "lo participante" al traspasar conscientemente mis funciones profesionales y compartir con los jóvenes que migran solos mi experiencia en aquellos aspectos que les resultaban útiles, más allá de lo administrativamente regulable. No se ha tratado, por tanto, de una técnica prediseñada, sino en una técnica sobrevenida. Cuando se decide permanecer "junto a" y no "enfrente de" los jóvenes, éstos, progresivamente, dejan de atribuir al investigador-técnico una posición de externo, de alguien relacionado con el Orden (protección de menores, educación, control migratorio, etc), pues hay una transacción activa de bienes en forma de saberes y emociones, impropia de los códigos instituidos en el Trabajo Social y su "distancia terapéutica", que lo transforman en un sucedáneo biomédico.

De esta manera, las notas iniciales en los cuadernos, procedentes de cierta observación flotante ${ }^{9}$, devinieron en una curiosidad más explícita, con conversaciones más centradas en el proceso migratorio, que no fueron grabadas pero sí registradas en notas. Durante todo el proceso, bien en encuentros provocados por los propios jóvenes, bien en la vida on line de las redes sociales, el contacto con intercambio ha sido una constante. Para algunos menores, se trataba de colaborar en "el libro"; para otros, una necesidad de sentir y valorizar como algo digno las historias de otros jóvenes a los que pedían que entrevistáramos, tanto como la suya propia ${ }^{10}$.

Evitábamos así el problema ético planteado por no confesar la posición de observador (Bergua, 2011: 166), pues la condición de "narrador" siempre se ha explicitado, y la costumbre de anotar, documentar o preguntar ha sido atribuida al investigador por los jóvenes desde que lo conocieron durante la práctica profesional.

8 "For me working as a social worker was not just a methodological tool of getting information; it was an ethical and moral choice" (Empez, 2009: 164).

${ }^{9}$ Bergua (2011: 172) atribuye ésta a Collette Péttonet: "mantenerse vacante y disponible sin fijar la atención en un objeto preciso, (...) dejándola flotar para que las informaciones penetren sin filtro". 
Tratábamos de eludir, en suma, el rechazo al paracaidista que otras observaciones podrían causar. Al contrario, hemos sido conscientes de que buscábamos el frotamiento. Cuando dos cuerpos se frotan, surge calor en ellos. Y eso los reconforta frente al frío exterior: el que acompaña a todo proceso migratorio o de ruptura con el entorno de origen; y el que, lamentablemente, envuelve algunos entornos profesionales del Trabajo Social. Además, surge también algo distinto, ese magnetismo o atracción que permite el ya mencionado, y creemos que tan necesario, intercambio. Antídoto homeopático contra la jerarquía, o al menos así lo hemos pensado.

"Pa cuando va a estar listo el libro? bueno, si puedo ayudarte en algo ya sabes aqui estoy. venga un saludo" (joven marroquí).

\section{A MODO DE CONCLUSIÓN}

Como propuesta final de reflexión, a la que podríamos añadir otras que quedan pendientes, como la necesidad del trabajo de campo multisituado (Empez, 2009: 161), la investigación nos ha planteado un dilema. ¿Qué podemos hacer entonces los que estamos estudiando y viviendo estos $u$ otros procesos migratorios "irregulares" como actores en uno de sus frentes (Bergua, 2007: 74)?

Nuestra investigación ha ayudado a poner de relieve las múltiples contradicciones de todos los actores profesionales, entre los que nos incluimos: que no soportan saber (Mai, 2011: 1238), que no quieren que se sepa, que quieren saber, que quieren y no pueden hacer, etc. Mas es en el magnetismo, en el no saber o razón común donde hemos encontrado el disfrute de comprender sin esfuerzo lo que la razón nos ocultaba. El perfil en las redes sociales nos ha acercado a los jóvenes. Las conversaciones en cafeterías como no-profesional nos han abierto matices antes ocultos por nuestro rol técnico. Lo cotidiano nos ha permitido ver, en definitiva, que tras el velo de la aparente uniformidad hay procedimientos, saberes y placeres muy diversos. Que fuera del Orden y dentro de él hay otro mundo donde reina la anarquía, formada por mezclas no jerárquicas, intratable (Bergua, 2007: 219). 
Algo siempre esperanzador para un Trabajo Social que reniegue de controlar.

Y, por otra parte, estos años también nos enseñan que el motivo final para no poder evitar "investigar" es que los profesionales sentimos una curiosidad innata por el mundo del que formamos parte. Por todo lo que rodea al Trabajo Social, pero sobre todo por todo lo que se le esconde. Afortunadamente, la verdadera recompensa a este instinto no es la comprensión como Poder, sino ese goce o "belleza" que Gregory Bateson (1998: 297) nos animaba a redescubrir.

Así hemos orientado nuestra investigación. Y por el camino, los antiguos menores ya son adultos, precarios que continúan su migración o surfean en la crisis junto al resto de nosotros. Ellos nos han devuelto estos años mucho más de lo que les hemos podido dar. A ellos y ellas se dedica este texto.

\section{REFERENCIAS BIBLIOGRÁFICAS}

BATESON, G. (1998). Pasos hacia una ecología de la mente. Buenos Aires: Lohlé-Lumen.

BAUMAN, Z. (2005). Vidas desperdiciadas. Barcelona: Paidós.

BERGUA, J.A. (2007). Lo social instituyente. Materiales para una sociología no clásica. Zaragoza: Prensas Universitarias.

BERGUA, J.A. (2011). Estilos de la investigación social. Técnicas, epistemología, algo de anarquía y una pizca de sociosofía. Zaragoza: Prensas Universitarias.

CABRERA, J. C. (2005). Acercamiento al menor inmigrante marroquí. Sevilla: Junta de Andalucía.

CASTORIADIS, C. (1983). La institución imaginaria de la sociedad. Volumen I. Marxismo y teoría revolucionaria. Barcelona: Tusquets.

DEBORD, G. (1958). Théorie de la dérive. Internationale Situationniste, 2, 19-23.

DELEUZE, G. (1999). Postadata sobre las sociedades de control. En C. Ferrer (coord.). El lenguaje libertario. Antología del pensamiento anarquista contemporáneo. Buenos Aires: Altamira.

DELEUZE, G. (2000). Mil mesetas. Valencia: Pretextos. 
EMPEZ, N. (2009). The fieldworker as a social worker: dilemma's in research with Moroccan Unaccompanied minors in Spain. En I. van Liempt y V. Bilger (ed.). The Ethics of Migration Research Methodology. Dealing with Vulnerable Immigrants. Eastbourne (Gran Bretaña): Sussex Academic Press.

GIMENO, Ch. (2012a). El imaginario de los menores que migran solos: aproximación desde las redes sociales. En VII Congreso Migraciones Internacionales en España. Euskal Herriko Univertsitatea: Bilbao.

GIMENO, Ch. (2013b). Trabajo Social y control migratorio. Tensiones en los Sistemas de Protección de Menores. Portularia, 13(2), 15-24.

GIMENO, Ch. (2013c). Menores que migran solos y sistemas de protección a la infancia, Zerbitzuan, 53, 109-122.

GIMENO, Ch. (2013d). Menores que migran solos: análisis de los expedientes de tutela administrativa en Aragón. Migraciones, 34, 139-175.

GIMENO, Ch. (2013e). Escenarios fuera de los sistemas de protección de menores: prostitución y redes de explotación. En "Políticas públicas y sociales en la encrucijada. Zaragoza: Prensas Universitarias de Zaragoza.

JIMÉNEZ, M. (2011). Intrusos en la fortaleza. Menores marroquíes migrantes en la Frontera Sur de Europa. Tesis doctoral. Universidad Autónoma de Madrid.

JUSTICIA DE ARAGÓN (2000 - 2010). Informes sobre la situación de los menores en Aragón. Zaragoza: Justicia de Aragón.

MAFFESOLI, M. (2004). El tiempo de las tribus. México: Siglo XXI.

MAI, N. (2011). Tampering with sex of angels: migrant male minors and young adults selling sex in the UE. Journal of Ethnic and Migration Studies, 8 (37), pp. 1237-1252.

MATEO, J. LI. (2010). Salud y ritual en Marruecos. Barcelona: Edicions Bellaterra.

MONTEROS, S. (2007). La construcción social de un nuevo sujeto migratorio: los menores migrantes marroquíes no acompañados. Tesis doctoral. Universidad Autónoma de Madrid. 
QUIROGA, V. (2005). Rutas de pequeños sueños. Los menores migrantes no acompañados en Europa [Informe comparativo]. Barcelona: Fundación Pere Tarrés.

QUIROGA, V. (2010). Sueños de bolsillo. Menores migrantes no acompañados en España. Barcelona: Unicef-Banesto.

SUÁREZ, L. (2008). La perspectiva transnacional en los estudios migratorios. Génesis, derroteros y surcos metodológicos. En J. García y J. Lacomba (eds.). La inmigración en la sociedad española. Una radiografía multidisciplinar. Barcelona: Edicions Bellaterra.

UNICEF (2009). Realidad jurídica y social de los menores extranjeros en España. Barcelona: Unicef.

VIRNO, P. (2003a). Virtuosismo y revolución. La acción política en la era del desencanto. Madrid: Traficantes de sueños.

VIRNO, P. (2003b). Gramática de la multitud. Para un análisis de las formas de vida contemporáneas. Madrid: Traficantes de Sueños. 